\title{
Conhecimento de profissionais e estudantes da área da saúde sobre as notificações de doenças compulsórias
}

\section{JulianadeOliveira}

G raduada em F isioterapia pelo C entro U niversitário de Barra M ansa - U BM

\section{PatrícialuaienedaCostaT eixeira}

M estrado em Ciências M édicas pela Universidade Federal Fluminense.

D ocente do C entro Universitário de B arra M ansa - UBM 


\section{Resumo}

Doença é qualquer enfermidade ou estado clínico que evidencie algum dano considerável aos seres humanos. A notificação compulsória se dá pela comunicação de ocorrência dos casos individuais, conjunto de casos ou surtos, suspeitos ou confirmados, da lista de agravos relacionados nas Portarias no 204 e Portaria 205, de fevereiro de 2016, do M inistério da Saúde e deve ser feita às autoridades sanitárias por profissionais de saúde ou qualquer cidadão, visando à adoção das medidas de controle pertinentes. Este trabalho teve como principal objetivo identificar o grau de conhecimento dos profissionais e estudantes da área da saúde acerca das doenças da notificação compulsória. Foram entrevistados 187 indivíduos da área da saúde acerca desse conhecimento, porém foram excluídos 50 participantes (27\%) por afirmarem não possuir conhecimento sobre as Doenças de Notificação Compulsória (DNC), assim, permaneceram no estudo 137 (73\%) indivíduos, sendo 78 (42\%) profissionais e 59 (32\%) estudantes da área da saúde. A o realizar a análise descritiva dos resultados deste estudo, pode-se ter a ideia do quão importante e relevante se faz a necessidade do conhecimento dos profissionais de saúde acerca da notificação de doenças compulsórias, o papel destes profissionais no processo de planejamento, ações de promoção e prevenção à saúde.

Palavras-chave: N otificação de doenças. Sistemas de informação em saúde. D oenças Compulsórias. Profissionais de saúde. 


\section{Abstract}

$D$ isease is any injury or clinical condition that shows any considerable harm to humans (M S, 2016). $M$ andatory reporting is given by the occurrence of communication of individual cases, number of cases or outbreaks, suspected or confirmed, the list of related diseases in O rdinances no. 204 and D ecree 205, February 2016, the M inistry of $\mathrm{H}$ ealth that should be made to sanitary authorities by health professionals or any citizen, with a view to adopting the pertinent control measures. This work has as main objective to identify the degree of knowledge of professionals and students of health about the selected compulsory report. We interviewed 187 individuals in the health field on this knowledge, but were excluded 50 subjects (27\%) by asserting has no knowledge on the Compulsory Notification Diseases (DNC) and remained in the study 137 (73\%) patients, 78 (42\%) professionals and 59 (32\%) students from the health area. In carrying out the descriptive analysis of the results of this study, one can have the idea of how important and relevant is the need for the knowledge of health professionals about the notification of compulsory diseases, the role of these professionals in the planning process, promotion actions and health prevention.

Keywords: $\mathrm{N}$ otification of illness. $\mathrm{H}$ ealth information systems. Compulsory Diseases. $\mathrm{H}$ ealth professionals. 


\section{Introdução}

D e acordo com M inistério da Saúde (2016), doença é qualquer enfermidade ou estado clínico que evidencie algum dano considerável aos seres humanos. Quando alguma doença se manifesta ou quando se tem alguma ocorrência em que possa causar doença é chamado de evento. Já quando ocorre dano físico, mental e social das pessoas devido a acidentes, intoxicações, drogas e lesões autoinfligidas ou heteroinfligidas é considerado agravo (M S,2016).

A notificação compulsória se dá pela comunicação de ocorrência dos casos individuais, conjunto de casos ou surtos, suspeitos ou confirmados, da lista de agravos relacionados nas Portarias no 204 e Portaria no 205, de fevereiro de 2016 do M inistério da Saúde, que deve ser feita às autoridades sanitárias por profissionais de saúde ou qualquer cidadão, visando à adoção das medidas de controle pertinentes. Como também a obrigatoriedade de registro de notificação para alguns eventos ambientais, doenças ou morte de determinados animais. O corrência de casos novos de doenças (transmissíveis ou não) ou agravo (inusitado ou não), com possibilidade de prevenção e controle pelos serviços de saúde, mostram que a população está sofrendo risco e pode representar ameaças à saúde e precisam ser detectadas e controladas ainda em seus estágios iniciais (M S, 2016).

O s procedimentos de notificação seguem a I nstrução N ormativa nํ 2, de 22 de novembro de 2005, que regulamenta as atividades da vigilância epidemiológica (VE) com relação à coleta, fluxo e a periodicidade de envio de informações da notificação compulsória de doenças por meio do Sistema de Informação de A gravos de N otificação (SINAN). 0 uso sistemático do SINAN, de forma descentralizada, colabora para a democratização da informação, possibilitando a todos os profissionais de saúde acesso à informação e tornando-as disponíveis para a comunidade. É um instrumento auxiliar para o planejamento da saúde, que define prioridades das intervenções, permitindo que sejam avaliados os impactos das mesmas (M S, 2005).

A Portaria 1.271 do M inistério da Saúde difere a notificação compulsória imediata e semanal. A primeira deve ser feita em até 24 horas a partir da descoberta do aparecimento da doença, evento ou agravo. Já a segunda, deve ser feita em até sete dias à secretaria de saúde do município em que o paciente está sendo atendido com a suspeita 
ou confirmação de doença ou agravo. Em ambos os casos, a autoridade que receber a notificação deverá informar às demais gestões dentro de 24 horas (M S, 2014).

o Sistema de Informações de Doenças de Notificação Compulsória, historicamente tem sido instrumento principal da VE, sendo primordial a preservação e aprimoramento constante, agregando avanços científicos e tecnológicos de cada período. Por isso as normas de notificação devem se adequar ao tempo e espaço (TEIXEIRA, et al., 1998), possibilitando aos municípios ou unidades federadas elaborarem listas complementares levando em conta a relevância local (HAM M A N N; LA GUARDIA, 2000).

No estudo realizado por M endes et al. (2000), a razão entre internações/ notificações evidenciou diferenças entre os dois sistemas de informações, como por exemplo, a subnotificação do Sistema de Internação Hospitalar (SIH) e Sistema $\mathrm{N}$ acional de V igilância E pidemiológica e as prováveis reinternações na rede hospitalar.

A epidemiologia nos serviços de saúde analisa a situação, planeja ações, atua na vigilância em saúde (epidemiológica, sanitária, nutricional, ambiental, do trabalho, etc.) analisa também o impacto de serviços, programas e tecnologias (M E N EZES, 2001).

É indispensável que 0 indivíduo atuante em porta de entrada dos estabelecimentos de saúde tenha conhecimento sobre notificação, para que não haja ocorrências não notificadas. Segundo Sousa (2012), a notificação compulsória é o elemento primordial para o desencadeamento de ações de vigilância em saúde e a subnotificação mascara 0 real estado de saúde da população. Diante do exposto, 0 presente estudo teve por objetivo verificar o real conhecimento dos profissionais e estudantes das áreas da saúde sobre N otificação C ompulsória.

\section{Metodologia}

E sta pesquisa teve início após aprovação no C omitê de É tica e Pesquisa, sob o CAAE no 68615717.9 .0000 .5255$.

Estudo descritivo, de caráter qualiquantitativo, em que participaram 187 profissionais e estudantes de ambos os sexos, atuantes na área da saúde, sendo estes: médicos, enfermeiros, fisioterapeutas, farmacêuticos, nutricionistas, psicólogos, veterinários, técnicos de enfermagem, biólogos, agentes sanitários e dentistas, que estudam em Instituições de ensino superior ou técnico, e profissionais atuantes em 
cidades localizadas na região do médio Paraíba, com faixa etária a partir de 18 anos, devidamente matriculadas no respectivo curso e que aceitaram participar do estudo conforme o T ermo de Consentimento Livre e E sclarecido (T CLE ).

Foram incluídos na pesquisa os indivíduos que aceitaram participar da pesquisa, assinando o TCLE, após explicações e retiradas de dúvidas sobre o questionário aplicado e excluídos os indivíduos que não responderam a todas as questões apresentadas.

Inicialmente, os participantes do estudo foram esclarecidos quanto ao TCLE, após a assinatura foi disponibilizado um questionário autoaplicável, produzido pelas autoras, contendo 13 questões objetivas, referentes à sua área de atuação, quanto ao entendimento desse indivíduo sobre notificação compulsória e como o mesmo avalia seu grau de conhecimento. 0 participante não foi autorizado a realizar qualquer tipo de consulta durante o procedimento e, ao término, foram sanadas as dúvidas pertinentes.

A pós a coleta, os dados foram exportados e analisados pelo M icrosoft O ffice Excel para melhor leitura dos resultados.

\section{Resultados}

Participaram do estudo 187 indivíduos de ambos os gêneros, profissionais e estudantes da área da saúde de I nstituições de ensino superior ou técnicos e profissionais atuantes em cidades localizadas na região do médio Paraíba, com faixa etária a partir de 18 anos, porém, foram excluídos 50 participantes (27\%) por afirmarem não possuir conhecimento sobre as Doenças de Notificação Compulsória (DNC). Assim, permaneceram no estudo 137 (73\%) indivíduos, sendo 78 (42\%) profissionais e 59 (32\%) estudantes da área da saúde (Figura 1).

O quantitativo dos entrevistados por área de atuação encontra-se descrito no gráfico 1 e as respostas obtidas em cada questão através do questionário aplicado na Tabela 1. 


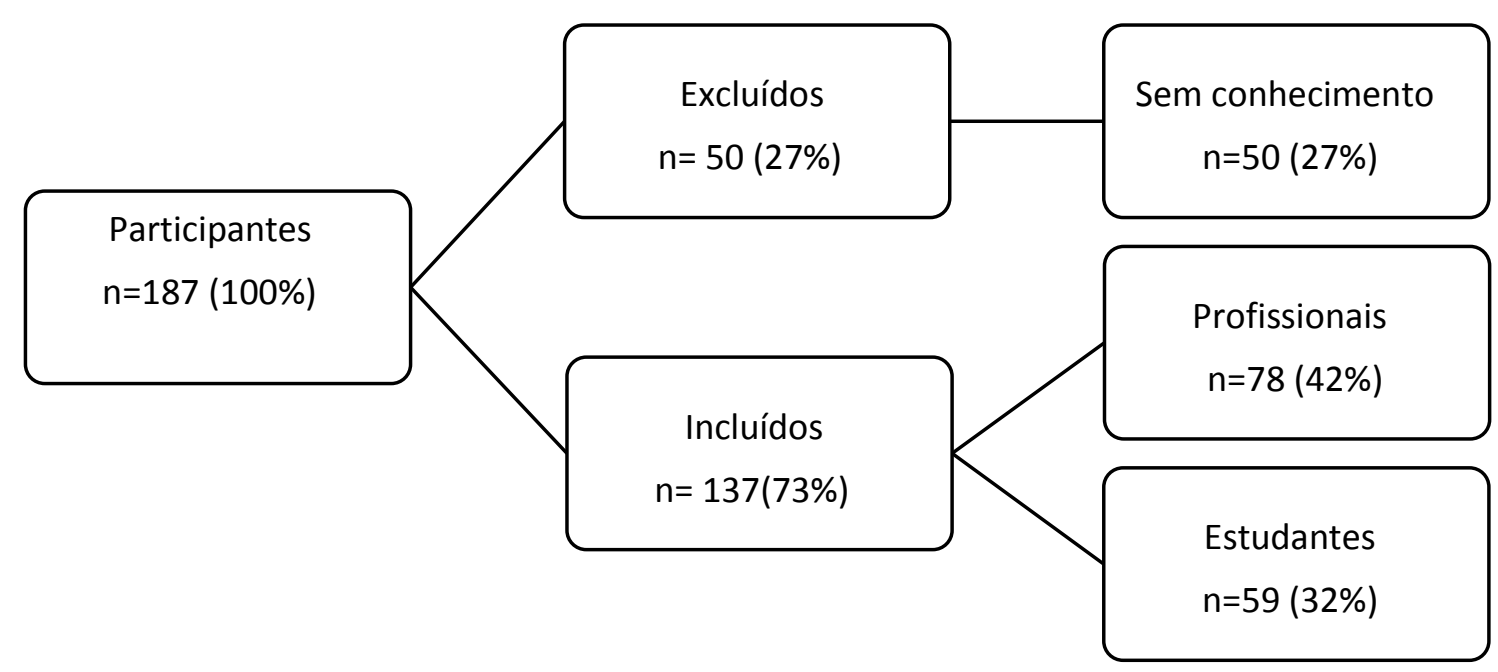

Figura 1. Perfil dos participantes

Fonte: A s autoras

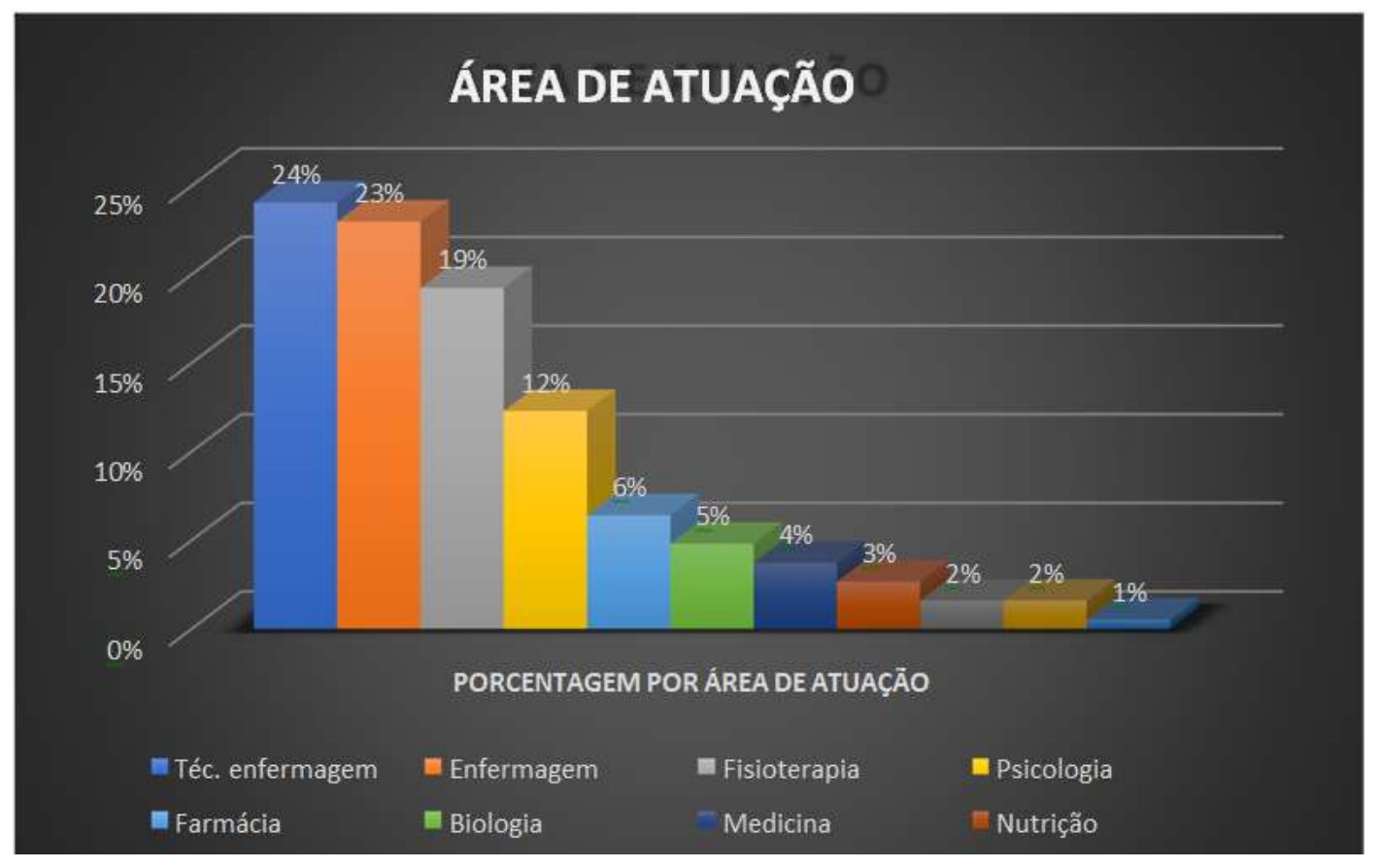

G ráfico 1 - T otal de entrevistados por área de atuação 
T abela 1 - R espostas obtidas pelo questionário

\begin{tabular}{|c|c|c|}
\hline Sabe o que são doenças de notificação compulsória? & $\mathrm{N}$ & $\%$ \\
\hline Sim & 111 & $59 \%$ \\
\hline N ão & 76 & $41 \%$ \\
\hline \multicolumn{3}{|c|}{ Como você avalia seu grau de conhecimento sobre as D N C? } \\
\hline N enhum & 50 & $27 \%$ \\
\hline Pouco & 54 & $29 \%$ \\
\hline Razoável & 57 & $30 \%$ \\
\hline Bom & 26 & $14 \%$ \\
\hline \multicolumn{3}{|l|}{ Q uais são as doenças de notificação compulsória? } \\
\hline Botulismo & 53 & $28 \%$ \\
\hline D ifteria & 77 & $41 \%$ \\
\hline A cidente vascular encefálico & 9 & $5 \%$ \\
\hline D engue & 97 & $52 \%$ \\
\hline C âncer de mama & 11 & $6 \%$ \\
\hline C âncer de próstata & 7 & $4 \%$ \\
\hline Febre maculosa & 67 & $36 \%$ \\
\hline M eningite & 65 & $35 \%$ \\
\hline Lombalgia & 9 & $5 \%$ \\
\hline H epatites virais & 52 & $28 \%$ \\
\hline D oença coronariana & 1 & $1 \%$ \\
\hline HIV & 84 & $45 \%$ \\
\hline Coqueluche & 62 & $33 \%$ \\
\hline Violência doméstica & 35 & $19 \%$ \\
\hline A cidentes de trabalho & 28 & $15 \%$ \\
\hline \multicolumn{3}{|c|}{ A comunicação de notificação pode ser realizada por qualquer cidadão? } \\
\hline Sim & 83 & $44 \%$ \\
\hline $\mathrm{N}$ ão & 49 & $26 \%$ \\
\hline $\mathrm{N}$ ão possui conhecimento & 56 & $30 \%$ \\
\hline
\end{tabular}


T abela 1 - Respostas obtidas pelo questionário (continuação)

\begin{tabular}{llc}
\hline O nde a notificação é realizada? & 56 & $30 \%$ \\
\hline R edes públicas & 56 & $30 \%$ \\
R edes privadas & 7 & $4 \%$ \\
A mbas & 76 & $41 \%$ \\
$N$ ão possui conhecimento & 46 & $25 \%$
\end{tabular}

Para onde deve ser feito o encaminhamento da notificação de uma doença ou agravo?

H ospitais

UBS

UPA

SM S

$\mathrm{N}$ ão possui conhecimento

Q uais os tipos de notificação compulsória?

Imediata

A nual

Semanal

$M$ ensal

$\mathrm{N}$ ão possui conhecimento

$37 \quad 20 \%$

$7 \quad 4 \%$

$0 \quad 0 \%$

$10154 \%$

$9450 \%$

$94 \quad 50 \%$

$32 \%$

$26 \quad 14 \%$

$169 \%$

$6434 \%$

\begin{tabular}{llll}
\hline Q ual o tempo máximo para a notificação imediata? & & \\
\hline 6 horas & 16 & $9 \%$ \\
12 horas & 15 & $8 \%$ \\
24 horas & 59 & $32 \%$ \\
36 horas & 8 & $4 \%$ \\
N ão possui conhecimento & 81 & $43 \%$ \\
\hline Como se faz a notificação? & & \\
\hline Pela suspeita de casos intra e extra hospitalares não confirmados por exames & 72 & $39 \%$ \\
A pós exames específicos para confirmação de casos de doença ou agravo & 57 & $30 \%$ \\
Busca ativa & 18 & $10 \%$ \\
Não possui conhecimento & 55 & $29 \%$ \\
\hline
\end{tabular}


T abela 1 - Respostas obtidas pelo questionário (continuação)

\begin{tabular}{lll}
\hline A notificação é obrigatória? & & \\
\hline Sim & 119 & $64 \%$ \\
N ão & 13 & $7 \%$ \\
N ão possui conhecimento & 54 & $29 \%$ \\
\hline Q ual sistema para registro das notificações em todo território nacional? & & \\
\hline SIN A N & 77 & $41 \%$ \\
SE RE & 0 & $0 \%$ \\
SRE P & 6 & $3 \%$ \\
SN C & 9 & $5 \%$ \\
N ão possui conhecimento & 95 & $51 \%$ \\
\hline L egenda: & & \\
UBS - Unidade Básica de Saúde & & \\
UPA - Unidade de Pronto A tendimento & & \\
SM S - Secretaria M unicipal de Saúde & & \\
SIN AN - Sistema de Informação de A gravos de N otificação & & \\
SE RE- sigla aleatória escolhida sem significado espećíico & & \\
SREP - Sistema de Registro Eletrônico de Ponto & & \\
SN C - Sistema N ervoso Periférico & &
\end{tabular}

\section{Discussão}

Conforme a resolução n. 287 (08/10/1998), faz-se necessário a participação de al guns profissionais na contribuição ativa para o C onselho N acional de Saúde (C NS), na consolidação das notificações junto ao Sistema Único de Saúde do Brasil (SUS). D essa forma, foi recrutado para este estudo um grupo de profissionais variados da área da saúde a fim de analisar o grau de conhecimento dos mesmos, pois a falta desse, do encaminhamento de fluxo reduzido às autoridades epidemiológicas, da certeza da notificação por outro profissional e também pela mudança da definição de caso, pode acarretar na baixa de notificação (CERR O N E; C A R M O , 2015).

Neste estudo, observou-se que $59 \%$ dos entrevistados relataram obter conhecimento acerca da definição de D N C, o que está de acordo com o estudo de Souza et al. (2012), o qual apresentou em sua maioria um bom conhecimento. A o avaliar 0 
grau desse conhecimento, 27\% da amostra responderam não apresentar qualquer tipo de entendimento sobre o assunto, sendo necessária a exclusão dos mesmos na análise dos resultados. Assim, dos participantes incluídos, 29\% expressaram pouco conhecimento, $30 \%$ razoável e $14 \%$ bom.

Grande parte dos entrevistados apontou a Dengue como sendo uma DNC $(52 \%)$, esse resultado pode ser explicado por essa patologia se tratar de um agravo relevante, mais noticiado e de grande incidência no Brasil, devido às características climáticas favorecerem a proliferação do vetor (aedes aegypti) e pela ineficácia das ações preventivas (BOH M et al., 2016).

No presente estudo, $45 \%$ identificaram a Infecção pelo Vírus da Imunodeficiência Adquirida (HIV/AIDS) como sendo doença de notificação compulsória. Brito et al. (2001) aponta que o conhecimento sobre HIV se baseia em estimativas estabelecidas, geralmente, pela aplicação de modelos matemáticos, fundamentados no número de casos notificados de AID S, nos resultados de estudos de soroprevalência em populações-sentinelas, e em inquéritos sorológicos pontuais em populações específicas, de baixo e alto risco para a exposição ao H IV .

A Difteria (TD) teve $41 \%$ de percentual nesta pesquisa, compreende-se como uma doença infecciosa de importância nos países do T erceiro $M$ undo, rara quando coberturas vacinais abrangem mais de $80 \%$ da população, frequentemente a ocorrência da doença em áreas com baixas condições sanitárias e socioeconômicas, em que a aglomeração de indivíduos é maior. C omumente, estas áreas apresentam baixa cobertura de vacinação e, por isso, não é obtido impacto no controle da disseminação da TD ( M S, 2016), a proteção contra a D ifteria pode ser realizada pela vacinação com o toxoide diftérico (e.g. DTP e outras combinações contendo o componente diftérico), mas não com antígenos somáticos e seu nível de imunidade reduz ao final da infância e adolescência, dependendo do calendário vacinal e da reserva de Corynebacteriumdi phtheriae na população (O L IVEIRA et al., 2011.)

A pesar dos acidentes de trabalho e da violência doméstica fazerem parte das DNC, somente $15 \%$ dos participantes optaram pela primeira, e 19\% pela segunda, sendo as assertivas menos marcadas. E mbora o Sistema de Informação de Saúde (SIS) seja moderno, os acidentes de trabal ho necessitam melhores registros, devido sua grande subnotificação pelo SIN A N , presumindo-se que a correta notificação seria uma maneira de proteção e prevenção ao trabalhador (G A L D IN O ; SA N T A N A ; FERRITE , 2012). 
Q uanto à Violência D oméstica, o M inistério da Saúde (M S) a aponta como grande parte de internação hospitalar e terceira maior causa de óbito no país, impactando diretamente na condição de saúde e qualidade de vida da população, assim sua abordagem e registro é recente e atualmente obrigatório, de acordo com o SINAN (VELOSO et al., 2013).

A o questionar se a notificação pode ser realizada por qualquer cidadão, $44 \%$ disseram que sim, $26 \%$, que não e $30 \%$ afirmaram não ter conhecimento. Segundo a portaria 204 de17 de fevereiro de 2016, Capítulo II, §3ㅇ, a comunicação de doença, agravo ou evento de saúde pública de notificação compulsória pode ser realizada à autoridade de saúde por qualquer cidadão que deles tenha conhecimento, sendo assim, tal afirmativa corrobora com o estudo, pois a maior parte dos indivíduos respondeu a este item do questionário corretamente.

Segundo o Guia de Vigilância Epidemiológica do M inistério da Saúde, a notificação compulsória é compreendida em: imediata (realizada até 24 horas), semanal (em até 7 dias), negativa (semanal, porém não havendo nenhuma identificação de agravo ou doença na semana epidemiológica, deve-se informar em um formulário do SINAN, encaminhando-o ao serviço de saúde pública) e sentinela (estratégia de vigilância de morbidade, mortalidade ou agentes etiológicos de interesse para a saúde pública, com participação facultativa).

No questionário do atual estudo, foram dispostas as seguintes opções quanto ao tipo de notificação: imediata, anual, semanal e mensal, tendo como respostas respectivamente $50 \%, 2 \%, 14 \%$ e $9 \%$, considerando um resultado positivo, pois as assertivas com a maior porcentagem são as opções corretas; apesar de 34\% não apresentarem conhecimento. Já ao tratar do tempo máximo para a notificação ser considerada imediata, 39\% dos indivíduos responderam corretamente ( 24 horas), ainda que $43 \%$ não possuíam conhecimento sobre a afirmativa.

A pesar de o SINAN constituir-se um instrumento auxiliar de planejamento e possibilitar o acesso à informação de todos os profissionais de saúde e da comunidade, observou-se, no estudo, que mesmo $41 \%$ dos participantes relatando-o como o sistema de registros, a maior parte dos profissionais (51\%) o desconhece.

Como disposto no Guia de Vigilância E pidemiológica, sabe-se que as DNC, como o próprio nome já diz, são de notificações obrigatórias, independentemente do tipo de notificação. $\mathrm{N}$ a pesquisa, ao serem questionados sobre tal obrigatoriedade, com 
exceção dos que relataram não possuir conhecimento (29\%), 64\% responderam de forma correta e 7\% disseram que não.

0 desempenho da Vigilância em Saúde deve adaptar-se aos modelos de gestão dos serviços públicos com abrangência aos serviços privados, integrando todos os níveis de atenção à saúde: primária, secundária e terciária (B O C A T T O , 2012), dessa forma, quanto ao questionamento do local de notificação; no estudo, 41\% responderam de forma exata (ambas), apesar de 25\% não terem conhecimento; 30\% relataram somente a rede pública e 4\% somente a privada. E ssa notificação deve ser encaminhada a qualquer serviço de saúde, porém, o maior percentual foi que esta deve ser feita diretamente na secretaria municipal de saúde (54\%).

$\mathrm{Na}$ questão 11, ao indagar-se como deve ser feita a notificação, 39\% dos participantes relataram que a notificação deve ser feita pela suspeita de casos intra e extra-hospitalares não confirmados por exames; 30\% acreditam que deve ser feita após exames específicos para confirmação de casos de doença ou agravo e $29 \%$ relataram não possuir conhecimento. Q ualquer das opções escolhidas pode ser considerada, entretanto, a busca ativa foi a menos marcada (10\%). 0 princípio de busca ativa pode ser a característica mais acentuada das práticas itinerantes, presente desde o sanitarismo campanhista, seja por busca de lugares insalubres de emanação de miasmas, de vetores ou de pessoas contaminadas, sempre na luta contra as patologias contagiosas (JUN IOR; NO G UEIRA, 2002; LIM A , 2002 apud LE M KE , 2010).

De acordo com o Art. 30 "A notificação compulsória é obrigatória para os médicos, outros profissionais de saúde ou responsáveis pelos serviços públicos e privados de saúde, que prestam assistência ao paciente, em conformidade com 0 art. 8ㅇda L eino 6.259, de 30 de outubro de 1975 e com oArt 14 que diz que "a inobservância das obrigações estabelecidas na presente L ei constitui infração da legislação referente à saúde pública, sujeitando o infrator às penalidades previstas no D ecreto-lei nำ785, de 25 de agosto de 1969, sem prejuízo das demais sanções penais cabíveis”. Ainda no âmbito legal, encontramos a citação da omissão de N otificação de D oença, apresentada no A rt. 269 do código penal (CP): D eixar o médico de denunciar à autoridade pública doença cuja notificação é compulsória: Pena detenção, de 6 (seis) meses a 2 (dois) anos, e multa. Sendo a assim, podemos dimensionar a responsabilidade do profissional de saúde e comunidade na disseminação, transmissão e descontrole das doenças de notificação, pelo simples fato da omissão dos casos à autoridade de saúde. 


\section{Considerações Finais}

A o realizar a análise descritiva dos resultados deste estudo, pode-se ter a ideia do quão importante e relevante se faz a necessidade do conhecimento dos profissionais de saúde acerca da notificação de doenças compulsórias, do papel destes profissionais no processo de planejamento e das ações de promoção e prevenção à saúde, conforme as referências encontradas.

Sendo o SINAN o sistema de informação para registro das notificações de doenças compulsórias em todo o território nacional, esses dados são aproveitados e encaminhados ao M inistério da Saúde e disponibilizados quando há necessidade de identificação de suspeitas e casos de doenças, eventos e agravos que devem ser levados a conhecimento internacional, dessa forma, o profissional, durante a assistência à população, ao se deparar com situações que o levem a identificação de alguma DNC, deve realizar a notificação e encaminhá-la à E pidemiologia de sua cidade por meio de formulário próprio, com a semana epidemiológica devidamente identificada. Já o cidadão que tiver conhecimento de doença, deve se dirigir a qualquer profissional e/ou estabelecimento de saúde e este se encarrega de direcionar a informação ao órgão pertinente, estando sujeito a penalidades constitucionais, caso essa notificação não seja realizada.

No estudo atual, ao observar as respostas no geral, notou-se certo equilíbrio, já que os participantes ou marcaram desconhecimento do assunto tratado e abandonaram 0 questionário ou, de acordo com o grau de conhecimento relatado, responderam coerentemente as opções. H ouve também alguma dificuldade na fundamentação e afirmação das questões relacionadas ao assunto, visto que estudos voltados para esse conhecimento ainda é limitado e reduzido, assim, sugere-se abordagem do assunto e estudos futuros voltados para a notificação.

\section{Referências}

B O C CA T T O, M arcia. Vigilância em saúde. São Paulo: U nifesp, 2012. 
BÖHM, Andrea Wendt et al. Dengue incidence trend in Brazil, 20022012. E pidemiologia eServiços de Saúde, v. 25, n. 4, p. 725-733, 2016.

BRIT O , A na M aria de et al. A ID S e infecção pelo H IV no B rasil: uma epidemia multifacetada. 2001. R evista da Sodedade B rasileira de M ediana T ropical, 34(2): 207-217, mar-abr, 2001.

CERRO N I, M atheus de Paula; CA R M O, E duardo $\mathrm{H}$ age. M agnitude das doenças de notificação compulsória e aval iação dos indicadores de vigilância epidemiológica em municípios da linha de fronteira do Brasil, 2007 a 2009. E pidemiologia eServiços de Saúde, v. 24, n. 4, p. 617-628, 2015.

DE O LIVEIRA, A lexandre A lves de Souza et al. D ifteria pelo Corynebacteriumulcerans: uma zoonose emergente no B rasil e no mundo. R evista de SaúdePública, v. 45, n. 6, p. 1176-1191, 2011.

G A L D IN O , A driana; SA N T A N A, Vilma Sousa; FE R RITE, Silvia. Os Centros de R eferência em Saúde do T rabalhador e a notificação de acidentes de trabalho no B rasil. UniversidadeF ederal da Bahia, 2012.

H A M M A N N , E dgar M erchán; L A G U A RD IA , Josué. R eflexões sobre a vigilância epidemiológica: mais além da notificação compulsória. I nformeE pidemiológico do SU S, v. 9, n. 3, p. 211-219, 2000.

http://bvsms.saude.gov.br/bvs/saudelegis/gm/2016/prt0204_17_02_2016.html. A cesso em: 12/11/2017.

http://notificacao.pbh.gov.br/. A cesso em: 14/02/2017.

http://www.planalto.gov.br/ccivil_03/leis/L 6259.htm. A cesso em: 18/12/2017.

http://www.saude.mppr.mp.br/pagina-261.html. A cesso em: 18/12/2017.

LE M KE, Ruben A rtur; SIL VA, Rosane A zevedo N eves da. A busca ativa como princípio político das práticas de cuidado no território. E studos e Pesqui sas em Psi cologia, v. 10, n. 1, 2010. 
M E N DES, A ntônio da C ruz G ouveia et al. A valiação do Sistema de Informações H ospitalaresSIH /SUS como fonte complementar na vigilância e monitoramento de doenças de notificação compulsória. I nforme E pidemi ológico do SU S, v. 9, n. 2, p. 6786, 2000.

M E N E ZE S, A .M .B. N oções básicas de epidemi ologia. E pidemi ologia das doenças respiratórias. V. 1, p.1-25, 2001.

M IN ISTÉ RIO D A SA ÚD E . Guia deVigilânda E pidemiológica. 8a edição, Brasília, D F, 2016.

PORTAL BRA SIL : Sistema de informação de agravos de notificação SIN AN . D isponível em: $<w$ ww. portalsinan.saude.gov.br/>. A cesso em:14/02/17

SO USA, Selônia Patrícia O liveira et al. C onhecimento sobre doenças e agravos de notificação compulsória entre profissionais da E stratégia Saúde da Família no município de T eresina, estado do Piaú, B rasil-2010. E pidemiologia eServiços de Saúde, v. 21, n. 3, p. 465-474, 2012.

TE IXE IRA , M aria da G lória et al. Seleção das doenças de notificação compulsória: critérios e recomendações para as três esferas de governo. I nforme epidemi ológico do SU S, v. 7, n. 1, p. 7-28, 1998.

VEL OSO, M ilene M aria X avier et al. N otificação da violência como estratégia de vigilância em saúde: perfil de uma metrópole do B rasil. Ciência \& Saúde Coletiva, v. 18, n. 5, 2013. 